\title{
CONDITIONS FOR EXACT CAVALIERI ESTIMATION
}

\author{
Mónica Tinajero-Bravo ${ }^{1}$, Guillermina Eslava-GómeZ² AND Luis M. \\ CRUZ-ORIVE ${ }^{\bowtie, 3}$
}

${ }^{1}$ Graduate studies in Mathematics, National Autonomous University of Mexico (UNAM), Ciudad Universitaria, 04510, D.F., Mexico; ${ }^{2}$ Department of Mathematics, Faculty of Sciences, National Autonomous University of Mexico (UNAM), Ciudad Universitaria, 04510, D.F., Mexico; ${ }^{3}$ Department of Mathematics, Statistics and Computation, Faculty of Sciences, University of Cantabria, Avda. Los Castros s/n, E-39005 Santander, Spain. e-mail: tinajerobm@gmail.com, eslava@matematicas.unam.mx, luis.cruz@unican.es (Received October 25, 2013; revised January 20, 2014; accepted March 19, 2014)

\begin{abstract}
Exact Cavalieri estimation amounts to zero variance estimation of an integral with systematic observations along a sampling axis. A sufficient condition is given, both in the continuous and the discrete cases, for exact Cavalieri sampling. The conclusions suggest improvements on the current stereological application of fractionator-type sampling.
\end{abstract}

Keywords: Murthy-Gundersen arrangement, order statistics, smooth fractionator, symmetric population, systematic sampling, zero variance estimation.

\section{INTRODUCTION}

Cavalieri sampling, namely systematic sampling along an axis, is highly efficient to estimate the population total whenever the measurement function satisfies certain conditions. In extreme cases the estimation may be exact, that is, the estimation variance may be zero. A trivial example arises when the measurement function is constant and the number of observations is a fixed integer (see, for instance, Fig. 2a in Mattfeldt, 1989). As a second example, sampling a fixed even number of Cavalieri observations along the base of an isosceles triangle will always yield the exact area of the triangle (see Fig. 2f in Mattfeldt, 1989). Such properties have a natural counterpart in the discrete case, namely whenever the population consists of a finite number of units. Murthy $(1967$, p. 165) considers a finite population in which the relevant values associated with the units follow a linear trend. He then defines "balanced systematic sampling" (bss), and notes that, if the population and the sample sizes are fixed even integers, then the estimation of the population total is exact. Actually, bss was rediscovered in a stereology context by Gundersen (2002), who called it the "smooth fractionator" design. Under the smooth fractionator arrangement a population following the linear trend is rearranged into a pattern analogous to an isosceles triangle, hence Murthy's observation.

In this note a sufficient condition is given in the continuous and discrete cases for Cavalieri estimation to be exact. A simple procedure is given to construct measurement functions satisfying such condition. It is also shown that the smooth fractionator arrangement of any such population will also yield exact Cavalieri estimation.

A different problem is how to arrange an arbitrary population so that the variance of the Cavalieri estimator is minimal ("best possible arrangement", see Fig. 2 in Gundersen, 2002). Intents pioneered by Sethi (1965) suggest that no general procedure may exist. Nonetheless the results given here suggest simple recommendations (see Discussion) that may help in a general case.

\section{PRELIMINARIES}

The basic stereological problem considered here is to estimate the volume of a fixed three dimensional subset from parallel systematic sections a constant distance apart, namely from planar Cavalieri sections. In this case the measurement function is section area.

In the continuous case the general target parameter is the integral

$$
Q=\int_{-\infty}^{\infty} f(x) \mathrm{d} x
$$

where $f: \mathbb{R} \rightarrow \mathbb{R}^{+}$is a bounded non-random function, called the measurement function, which is square integrable in a bounded domain $D \subset \mathbb{R}$ (consisting of finite union of non empty closed intervals on a sampling axis), and vanishes outside $D$. Without loss of generality, in the sequel it is convenient to 
assume that $D=[0,2 h], h>0$. The standard unbiased estimator of $Q$ is the Cavalieri estimator,

$$
\widehat{Q}=T \sum_{k \in \mathbb{Z}} f(z+k T),
$$

where $T>0$ is the sampling period, namely the constant distance between consecutive observations, whereas $z \sim \operatorname{UR}[0, T)$ is a uniform random variable in the interval $[0, T)$.

In the discrete case the target parameter is the total

$$
Q=\sum_{i=1}^{N} y_{i}
$$

of a population consisting of a finite number $N \in \mathbb{N}$ of units. The values associated with the units are,

$$
Y:=\left\{y_{1}, y_{2}, \ldots, y_{N}\right\}
$$

For convenience we set $y_{i}=0, i \notin\{1,2, \ldots, N\}$. The standard unbiased estimator of $Q$ is,

$$
\widehat{Q}=T \sum_{k \in \mathbb{Z}} y_{z+k T},
$$

where $T \in \mathbb{N}$ is the (finite) sampling period and $z$ is a uniform random integer from the set $\{1,2, \ldots, T\}$.

\section{CONTINUOUS CASE}

\section{MAIN RESULT (CONTINUOUS CASE)}

Consider the following special case of the Cavalieri estimator (Eq. 2) for which the sample size is a fixed integer $n$, namely,

$\widehat{Q}(z)=T \sum_{k=0}^{n-1} f(z+k T), T=\frac{2 h}{n}, n \in \mathbb{N}, z \sim \operatorname{UR}[0, T)$.

Besides, consider the following assumption,

$$
\text { C1: } n=2 m, m \in \mathbb{N} \text {, fixed, }
$$

that is, $n$ is a fixed even number. Then, the Cavalieri estimator may be written as

$Q_{m}(z)=T \sum_{k=0}^{m-1}\{f(z+k T)+f(z+k T+h)\}, T=\frac{h}{m}$.

Proposition 1. Under the assumption $\mathrm{C} 1$, a sufficient condition that $Q_{m}(z)=Q$ for each $m \in \mathbb{N}$ and all $z \in[0, T)$, is that $Q_{1}(z)=Q$ for all $z \in[0, T)$, namely,

$$
\begin{aligned}
\mathrm{C} 2: & f(z)+f(z+h)=2 \mu,(\text { constant }), \\
& \text { for all } z \in[0, h],
\end{aligned}
$$

where $\mu=Q /(2 h)$ is the mean value of $f(x)$ in the interval $[0,2 h]$.

Proof. From the definition (Eq. 8) and the condition $\mathrm{C} 2$, it trivially follows that,

$$
Q_{m}(z)=T \sum_{k=0}^{m-1} \frac{Q}{h}=\frac{h}{m} \cdot m \cdot \frac{Q}{h}=Q, \quad m \in \mathbb{N} .
$$

Remark 1. Under the assumption $\mathrm{C} 1$, the identity

$$
Q_{m}(z)=Q \quad \text { for all } z \in[0, h / m) \text {, for some } m>1,
$$

does not imply the condition C2: $Q_{1}(z)=Q$ for all $z \in[0, h)$ - thus, the condition $\mathrm{C} 2$ is sufficient but not necessary. As an example, consider the following measurement function,

$$
f(x)= \begin{cases}2 x, & x \in[0,1 / 4) \cup[1 / 2,3 / 4), \\ 3-2 x, & x \in[1 / 4,1 / 2) \\ 2-2 x, & x \in[3 / 4,1)\end{cases}
$$

It can be verified that $Q_{m}(z)=1=Q$ is constant for even $m$ and for all $z \in[0,1 /(2 m))$, whereas

$$
f(x)+f(x+1 / 2)= \begin{cases}4 x+1, & x \in[0,1 / 4), \\ 4-4 x, & x \in[1 / 4,1 / 2)\end{cases}
$$

is not.

\section{IMPLICATIONS (CONTINUOUS CASE)}

\section{Construction}

Proposition 1 suggests a construction method for a measurement function $f(x), x \in[0,2 h]$, which satisfies Eq. 9. Define a square integrable function $\varphi:[0, h] \rightarrow$ $[0, Q / h]$. Then,

$$
f(x)= \begin{cases}\varphi(x), & x \in[0, h], \\ Q / h-\varphi(x-h), & x \in(h, 2 h] .\end{cases}
$$

To provide an intuitive interpretation, suppose that $\varphi(x)>0, x \in[0, h]$. Then the graph of $\varphi(x)$ splits the rectangle $R:=[0, h] \times[0, Q / h]$ into two nonoverlapping regions $R_{-}$(lower region) and $R_{+}$(upper region), that is, $R=R_{-} \cup R_{+}, R_{-} \cap R_{+}=\emptyset$. Thus the region under the measurement function $f(x)$ is the union of $R_{-}$and a translate of the reflection of $R_{+}$ with respect to the upper side of $R$, see Fig. 1. If it is desired that $f(x)$ is continuous, then choose $\varphi(x)$ to be continuous in $[0, h]$ with $\varphi(h)=Q / h-\varphi(0)$, (Fig. 1c, d). 


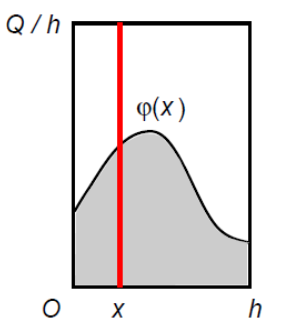

(a)

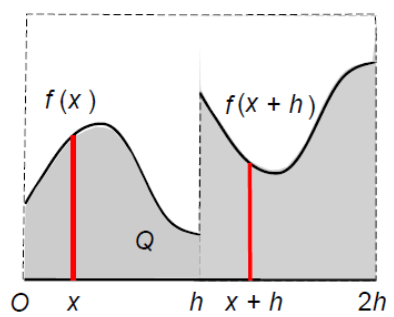

(b)

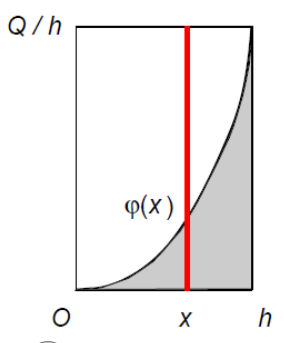

(c)

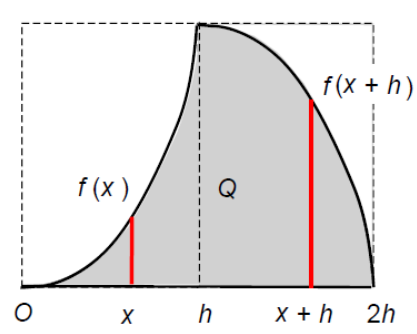

(d)

Fig. 1. (a), (b) Construction of a measurement function $f(x)$ - according to Eq. 14 - for which Cavalieri estimation is exact for any fixed even number of observations (red line segments in $(b)$ ). The target parameter $Q$, namely the grey area under the measurement function in (b), is equal to the area of the rectangle in (a). The sum of any pair of Cavalieri observations a distance $h$ apart in (b) is always equal to the height of the rectangle in (a), namely $Q / h$, see Eq. 9. (c), (d) Example of a continuous measurement function with the same property, see text.

\section{Symmetric measurement function}

Proposition 2. Suppose that the measurement function satisfies the following symmetry property,

$$
f(x)=f(2 h-x), \quad x \in[0,2 h] .
$$

Then, the condition $\mathrm{C} 2$ (Eq. 9), is a necessary and sufficient condition that the measurement function satisfies the additional symmetry property:

$$
f(h / 2)-f(x)=f(h-x)-f(h / 2), \quad x \in[0, h] .
$$

Proof. First we prove the sufficiency namely, if the conditions (Eq. 9) and (Eq. 15) hold, then Eq. 16 holds. The identity (Eq. 15) implies $f(h-x)=f(h+x)$, $x \in[0, h]$, which together with condition C2 yields $f(x)+f(h-x)=2 \mu, x \in[0, h]$. Thus $f(h / 2)=\mu$ and therefore $f(x)+f(h-x)=2 f(h / 2), x \in[0, h]$, which is Eq. 16. Now we prove the necessity, that is, if Eq. 15 and Eq. 16 hold, then Eq. 9 holds. Substitution of $f(h-x)$ with $f(h+x)$ in the r.h.s. of Eq. 16 yields $f(x)+f(h+x)=2 f(h / 2)$. Integration of both

sides of the preceding identity from $x=0$ to $x=h$, yields $Q=2 h f(h / 2)$, whereby $f(h / 2)=\mu$, and the identity (Eq. 9) follows.

Thus, under the assumption $\mathrm{C} 1$ (Eq. 7), if a measurement function satisfies the two symmetry properties (Eqs. 15 and 16), then $\mathrm{C} 2$ holds, and by Proposition 1 it will yield exact Cavalieri estimation.

Example 1. The ordinary symmetry property (Eq. 15) alone does not warrant exact Cavalieri estimation. For instance, the measurement function

$$
f(x)= \begin{cases}x^{2}, & x \in[0,1), \\ (2-x)^{2}, & x \in[1,2],\end{cases}
$$

satisfies $f(x)=f(2-x), x \in[0,2]$ but not the condition (Eq. 16), and therefore it does not yield exact Cavalieri estimation.

Example 2. The following measurement function

$$
f(x)= \begin{cases}1+(x-1)^{3}, & x \in[0,2], \\ 1-(x-3)^{3}, & x \in(2,4],\end{cases}
$$

(see Fig. 2) satisfies the symmetry conditions (Eqs. 15, 16) and hence the condition (Eq. 9). Thus, any Cavalieri sample of a fixed even size will yield the target parameter $Q=4$ without error.

Further examples are considered in Ch. 4 of Tinajero-Bravo (2014).

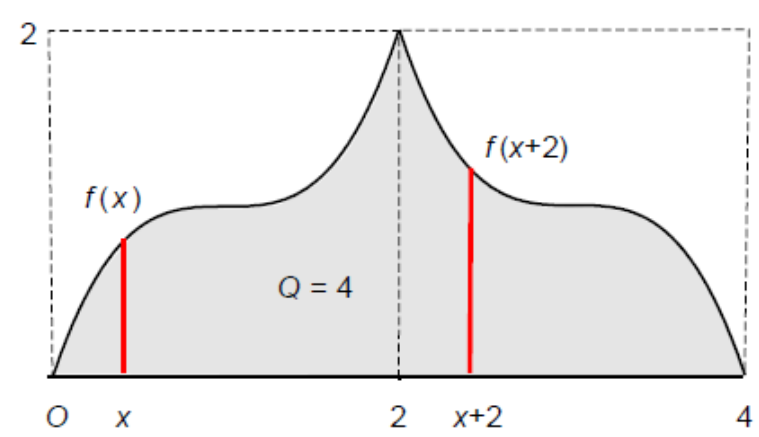

Fig. 2. Graph of the symmetric measurement function of Example 2. An even number of Cavalieri observations (red line segments) will always yield the target parameter $Q$ without error.

\section{DISCRETE CASE}

\section{MAIN RESULT (DISCRETE CASE)}

Consider Cavalieri sampling of the population $Y$ (Eq. 4), with the following assumptions,

$$
\begin{array}{ll}
\text { D1: } & n=2 m, m \in \mathbb{N}, \text { fixed, } \\
\text { D2: } & N=2 M, M \in \mathbb{N}, \text { fixed, } \\
\text { D3: } & T=M / m \in \mathbb{N} .
\end{array}
$$


Thus, both the sample and the population sizes are fixed even integers and the latter is multiple of the former. Note that, unlike the continuous case, $m$ does not necessarily take consecutive values $1,2, \ldots, N / 2$ for fixed $N$. Now the unbiased Cavalieri estimator (Eq. 5) may be written,

$$
\begin{aligned}
Q_{m}(z) & =T \sum_{k=0}^{m-1}\left\{y_{z+k T}+y_{z+k T+M}\right\}, \\
z & \sim \operatorname{UR}\{1,2, \ldots, T\} .
\end{aligned}
$$

Proposition 3. Under the assumptions D1-D3, a sufficient condition that $Q_{m}(z)=Q$ for each $m \in \mathbb{N}$ and all $z \in\{1,2, \ldots, T\}$, is that $Q_{1}(z)=Q$ for all $z \in\{1,2, \ldots, M\}$, namely,

$$
\text { D4: } \begin{array}{r}
y_{z}+y_{z+M}=2 \mu,(\text { constant }), \\
\text { for all } z \in\{1,2, \ldots, M\},
\end{array}
$$

where $\mu=Q /(2 M)$ is the mean value of $y_{x}, x=$ $1,2, \ldots, N$.

Proof. Analogous to the proof of Proposition 1.

Remark 2. Under the assumptions D1-D3, the condition D4 is not necessary for the identity $Q_{m}(z)=$ $Q$ to hold for each $m \in \mathbb{N}$ and for all $z \in\{1,2, \ldots, T\}$. As an example, consider the following discrete population,

$$
Y=\{6,6,3,12,8,4,1,3,11,5,7,6\} .
$$

Because $N=12$, conditions D1 and D3 imply that $m \in\{1,2,3\}$. It can readily be verified that $Q_{2}(z)=$ $Q_{3}(z)=72=Q$, whereas $Q_{1}(z) \neq Q$ for all $z \in$ $\{1,2, \ldots, 6\}$.

Remark 3. If conditions D1-D4 hold, then the population $Y$ consists of $M$ pairs of element values such that the sum of each pair is equal to $2 \mu$. An element of any such pair is called a 'match' of the other element in the pair, and the pair itself is called a 'matched pair'. The preceding assertion is easy to verify because, if there is a $k \in\{1,2, \ldots, N\}$ for which the element $y_{k}$ does not have a match in the population, then Eq. 21 fails to hold for $z=k$.

Remark 4. Under conditions D1-D4, any Cavalieri sample consists of $m$ matched pairs. This is easy to verify bearing in mind the form of the r.h.s. of Eq. 20 and the identity in Eq. 21.

\section{IMPLICATIONS (DISCRETE CASE)}

\section{Construction}

The rectangular construction idea proposed for the continuous case extends here with the proper adaptation. In practice the populations of interest are usually discrete, and therefore their elements are often easy to rearrange prior to sampling. Relevant tools in this context are the order statistics.

The order statistics $\left\{y_{(k)}, k=1,2, \ldots, N\right\}$ of the population $Y$ are the successive elements of the population resulting when the values $\left\{y_{k}, k=\right.$ $1,2, \ldots, N\}$ are sorted in non decreasing order of magnitude, namely,

$$
y_{(1)} \leq y_{(2)} \leq \cdots \leq y_{(N-1)} \leq y_{(N)} .
$$

Although Corollary 1 below is fairly direct, it is stated as such for convenience of exposition.

Corollary 1. If condition D4 holds, then the order statistics of the population $Y$ may be expressed as follows

$$
Y_{0}:=\left\{y_{(1)}, y_{(2)}, \ldots, y_{(M)}, 2 \mu-y_{(M)}, \ldots, 2 \mu-y_{(1)}\right\}
$$

Proof. For simplicity, and without loss of generality, suppose that the $N$ values from $Y$ are different. Then, in any matched pair one of the two element values is less than $\mu$, whereas the other is necessarily larger than $\mu$ because the sum of both matched values is $2 \mu$, see Remark 3. Thus the population consists of $M$ values less than $\mu$ - which are precisely the first $M$ elements of $Y_{0}-$ and another $M$ values larger than $\mu$, which are the last $M$ elements of $Y_{0}$.

\section{Symmetries (discrete case)}

If the identity (Eq. 21) holds, then the order statistics of the population $Y$ are symmetric about the population mean $\mu$, that is,

$$
\mu-y_{(k)}=y_{(N-k+1)}-\mu, \quad k=1,2, \ldots, M .
$$

In fact, Eq. 24 implies that

$$
y_{(N-k+1)}=2 \mu-y_{(k)}, \quad k=1,2, \ldots, M,
$$

which is equivalent to Eq. 25.

\section{The Murthy-Gundersen smoothing arrangement}

If the population $Y$ satisfies the property D2, (mid Eq. 19), then a Murthy-Gundersen arrangement (called the smooth fractionator arrangement in Gundersen, 2002) is,

$$
Y_{M G}:=\left\{y_{(1)}, y_{(3)}, \ldots, y_{(N-1)}, y_{(N)}, \ldots, y_{(4)}, y_{(2)}\right\} .
$$

Thus, $Y$ is first sorted in non decreasing order of magnitude to form the order statistics $Y_{O S}=$ $\left\{y_{(k)}, k=1,2, \ldots, N\right\}$. Let $Y_{o}, Y_{e}$ denote the two 
subsets of odd and even orders from $Y_{O S}$, respectively, and let the subset $Y_{e}^{r}$ denote the reverse of $Y_{e}$. Then choose among $Y_{M G}=\left\{Y_{o}, Y_{e}^{r}\right\}$, or its reverse $Y_{M G}^{r}$, with probability $1 / 2$.

Corollary 2. If the identity (Eq. 21) holds for the population $Y$, then the corresponding MurthyGundersen arrangement $Y_{M G}$ also satisfies the identity.

Proof. Write the identity (Eq. 27) as follows,

$$
\begin{aligned}
& Y_{M G}=\left\{y_{1}^{*}, y_{2}^{*}, \ldots, y_{N}^{*}\right\} \\
& y_{i}^{*}=y_{(2 i-1)}, i=1,2, \ldots M \\
& y_{i+M}^{*}=y_{(N-2 i+2)}, i=1,2, \ldots M .
\end{aligned}
$$

By replacing $k$ with $2 i-1$ on both sides of Eq. 26, the required identity $y_{i}^{*}+y_{i+M}^{*}=2 \mu$ is obtained.

Corollary 3. Suppose that the order statistics of the population $Y$ are symmetric about $\mu$ (Eq. 25), the assumptions D1-D3 hold, and there is an arrangement of $Y$ such that, whenever $y_{(i)}$ belongs to a Cavalieri sample for any $i \in\{1,2, \ldots, N\}$, the order statistic $y_{(N-i+1)}$ also does. Then, the identity in Eq. 21 holds.

Proof. For $m=1$ the Cavalieri estimator (Eq. 20) reads,

$$
Q_{1}(z)=M\left(y_{z}+y_{z+M}\right), \quad z \in\{1,2, \ldots, M\} .
$$

Without loss of generality, suppose that the $N$ population values are different. For any fixed $z \in$ $\{1,2, \ldots, M\}$ there is a unique index $i \in\{1,2, \ldots, N\}$ such that $y_{z}=y_{(i)}$, whereby,

$$
Q_{1}(z)=M\left(y_{(i)}+y_{z+M}\right) .
$$

Corollary 3 states that, if $y_{(i)}$ belongs to a Cavalieri sample, then $y_{(N-i+1)}$ also does. Therefore,

$$
Q_{1}(z)=M\left(y_{(i)}+y_{(N-i+1)}\right) .
$$

Moreover, if the order statistics are symmetric about $\mu$, then the identity (Eq. 26) holds. Thus, $Q_{1}(z)=$ $2 \mu M$ which, equated to the r.h.s. of Eq. 29, yields the identity (Eq. 21).

Remark 5. The Murthy-Gundersen arrangement (Eq. 27), for instance, satisfies Corollary 3.

Example 3. The discrete population

$$
Y=\{3,1,4,2,7,9,6,8\}
$$

has even size $N=2 M=8$ and satisfies the identity (Eq. 21). Therefore, Cavalieri estimation of the population total $Q=40$ is exact for $n=2,4$, (namely for $T=4,2$ respectively). Note that $n=6$ is not eligible because $T=8 / 6$ is not an integer. The corresponding order statistics

$$
Y_{0}=\{1,2,3,4,6,7,8,9\}
$$

are symmetric about $\mu=5$, namely $5-1=9-5$, $5-2=8-5$, etc., Eq. 25. The two possible MurthyGundersen arrangements are,

$$
\begin{aligned}
& Y_{M G}=\{1,3,6,8,9,7,4,2\}, \\
& Y_{M G}^{r}=\{2,4,7,9,8,6,3,1\} .
\end{aligned}
$$

Note that both arrangements satisfy the identity (Eq. 21), and hence they also yield exact Cavalieri estimation (Corollary 2).

\section{DISCUSSION}

Propositions 1 and 3 constitute the core of this note - they establish sufficient conditions for Cavalieri sampling to yield the target parameter $Q$ without error for any even sample size from a population of even size. These results constitute a theoretical guideline only, because the identities (Eq. 9) and (Eq. 21), the construction (Eq. 14), etc., involve the unknown parameter $Q$. On the other hand, the Murthy-Gundersen arrangement (Eq. 27) involves the values $\left\{y_{k}\right\}$ of the target population $Y$, which are usually unobservable prior to sampling. Typically in stereology, the $k$ th sampling unit is an opaque block of material containing $y_{k}$ particles, which are not directly observable. Thus the mentioned arrangement has to be based on the apparent size of the blocks (Gundersen, $1986 ; 2002)$. The theory would apply exactly if $y_{k}$ was proportional to say the observable volume, or the weight $x_{k}$ of the $k$ th block. Failing this, exact estimation is hardly attainable in practice.

Notwithstanding the foregoing remarks, practical recommendations stemming from this note may be the following:

(i) Aim at an even population size $N$, that is, start with an even number of blocks.

(ii) Fix an even sample size $n$, ensuring that the sampling period $T=N / n$ is an integer.

The preceding two recommendations are likely to help in practice, but they are only speculative and do not need to reduce estimation variance in all cases.

Hitherto it has largely been advocated to start with a population whose values follow an approximately linear trend (i.e. consisting of a smooth gradation of block sizes, from rather small to fairly large) followed by a Murthy-Gundersen arrangement, with little concern about the sample size. In Fig. 3a from Cruz-Orive and Weibel (1981), a fixed sample size $n$ was advocated and ensured for Cavalieri sampling by adopting a distance $T=H / n$ between sections, where $H$ was the caliper length of the object, namely 
the orthogonal projected length of the object onto the sampling axis. Later, the influential paper of Gundersen and Jensen (1987) relaxed the restriction of fixing $n$, because $H$ may be difficult, or at least laborious, to measure in the laboratory. In fact, the target object is often embedded in agar (which is not transparent) prior to slicing, see for instance Michel and Cruz-Orive (1988). Unfortunately the latter strategy has prevailed also in the discrete case, and specially in the fractionator context, in which fixing $N$ and $n$ should usually be easy. This is strange because it is well known that allowing the sample size $n$ to be random increases the so called Zitterbewegung, which often constitutes an important contribution to the total estimation variance (Kiêu et al., 1999; GarcíaFiñana and Cruz-Orive, 2000).

The beneficial effect on the estimation accuracy of adopting even and fixed population and sample sizes would still be enhanced if the population consisted of "matched pairs" of blocks, (Remark 3), and if the Murthy-Gundersen arrangement was then used (Corollary 2). The jumps of the measurement function, or of its derivatives, also contribute to the variance (Kiêu et al., 1999; García-Fiñana and Cruz-Orive, 2004). However, in the ideal case in which $N, n$ are both even, the population consists of matched pairs, and the Murthy-Gundersen arrangement is adopted, the variance tends to vanish in spite of the presence of any jumps among consecutive population values in the arrangement. Fig. 1b illustrates this effect in the continuous case.

\section{ACKNOWLEDGMENTS}

The authors are grateful to an anonymous referee for a number of constructive remarks. MTB acknowledges a scholarship for graduate estudies of the Mexican National Council for Science and Technology (CONACYT). LMCO acknowledges support of the Ministry of Education and Science I+D Project MTM-2009-14500-C02-01.

\section{REFERENCES}

Cruz-Orive LM, Weibel ER (1981). Sampling designs for stereology. J Microsc 122:235-72.

García-Fiñana M, Cruz-Orive LM (2000). New approximations for the variance in Cavalieri sampling. J Microsc 199:224-38.

García-Fiñana M, Cruz-Orive LM (2004). Improved variance prediction for systematic sampling on $\mathbb{R}$. Statistics 38:243-72.

Gundersen HJG (1986). Stereology of arbitrary particles. A review of unbiased number and size estimators and the presentation of some new ones, in memory of William R. Thompson. J Microsc 143:3-45.

Gundersen HJG (2002). The smooth fractionator. J Microsc 207:191-210.

Gundersen HJG, Jensen EB (1987). The efficiency of systematic sampling in stereology and its prediction. J Microsc 147:229-63.

Kiêu K, Souchet S, Istas J (1999). Precision of systematic sampling and transitive methods. J Statist Plan Inf 77:263-79.

Mattfeldt T (1989). The accuracy of one-dimensional systematic sampling. J Microsc 153:301-13.

Michel RP, Cruz-Orive LM (1988). Application of the Cavalieri principle and vertical sections method to lung: estimation of volume and pleural surface area. J Microsc 150:117-36.

Murthy MN (1967). Sampling theory and methods. Statistical Publishing Society, Calcutta.

Sethi VK (1965). On optimum pairing of units. Sankhya 27: $315-20$.

Tinajero-Bravo M (2014). Varianza del estimador del total bajo muestreo sistemático de algunas poblaciones simétricas. Unpublished PhD Thesis, UNAM, Mexico. 